TITLE: STUDIES OF SLIDING FRICTION IN COMPRESSED COPPER

AUTHOR(S): J. E. HAMMERBERG

B. L. HOLIAN

S. J. ZHOU

SUBMITTED TO: APS TOPICAL CONFERENCE

SEATTLE, WA

AUGUST, 13-18, 1995

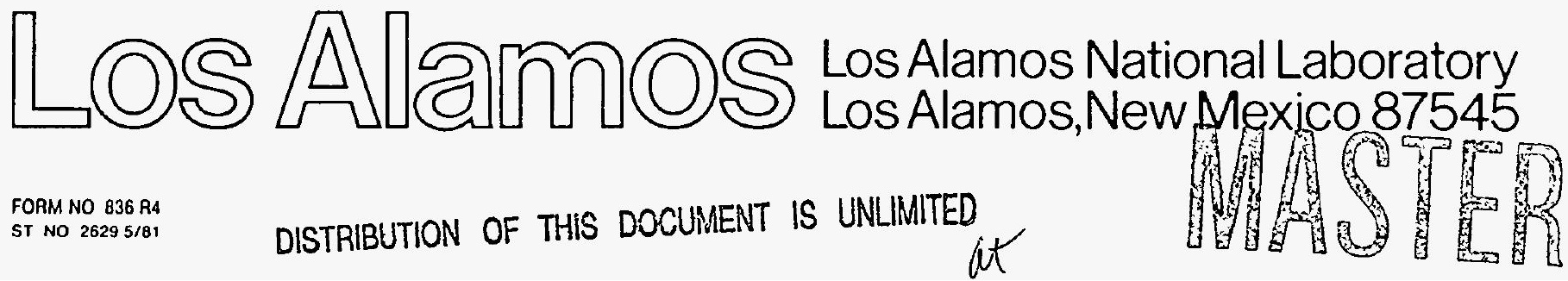




\section{DISCLAIMER}

Portions of this document may be illegible in electronic image products. Images are produced from the best available original document. 


\title{
STUDIES OF SLIDING FRICTION IN COMPRESSED COPPER
}

\author{
J.E.Hammerberg \\ Applied Theoretical and Computational Physics Division \\ B.L.Holian \\ Theoretical Division \\ S.J.Zhou \\ Center for Nonlinear Studies \\ Los Alamos National Laboratory \\ Los Alamos, NM 87545
}

We present the results of simulations for sliding copper interfaces for pressures in the kilobar range. The velocity dependence and density dependence are discussed and the evolution of plastic damage is described. Density dependent embedded atom model (EAM) potentials are used to describe the atomic interactions. Simulations were typically for $\mathrm{N}=65,000$ atoms and were carried out using molecular dynamics on massively parallel CM200 and CM5 platforms. A transition to a low friction state at high velocities is discussed.

\section{INTRODUCTION}

The nature of the average microscopic forces between two sliding metals determines the macroscopic evolution of sliding material interfaces. The physics of the macroscopic evolution in turn determines the constitutive laws relating components of forces at an interface which, for example, enter into materials continuum codes that model material flow and deformation. The same understanding is important for modeling wear mechanisms at interfaces. The low velocity, normal pressure behavior of surfaces has become increasingly accessible to very accurate measurement, due to the development of various atomic scale microscopies, e.g., STM, AFM. The situation is not at the same stage of development for high velocities and high densities. There are, however, in the latter situation numerical experiments at a similar level of refinement which can shed light on the microscopic physics of interfacial dynamics. In this paper we discuss a series of such simulations for sliding copper interfaces at high densities for velocities at significant fractions of the sound speed. The method we employ is molecular dynamics (MD) for systems of order
65,000 atoms on massively parallel computers, the CM5 and CM200. The following sections give a brief description of the theory of friction at high densities, our simulation method, the application to copper interfaces, and a discussion of the density and velocity dependence of the results of these simulations.

\section{FRICTIONAL FORCES AT HIGH DENSITIES}

For ductile metals, the adhesive model of asperity interactions is a reasonable model for deformation at a metal-metal interface. In this model, microscopic inhomogeneities (asperities) are in contact under the local load required for mechanical stability at shear strains sufficient to initiate plastic flow. Under such circumstances the tangential force, averaged over the evolving asperity distribution, is proportional to the effective area of contact and the flow stress of the weaker of the two materials forming the interface

$$
\left\langle F_{\text {tang }}\right\rangle=A_{e} \tau
$$


In this mean field type of picture both effective area, $A_{e}$, and flow stress, $\tau$, are functions of temperature $T$, density $\rho$, plastic strain $\psi$, plastic strain rate $\psi$, relative velocity of the interfaces and asperity distribution function. Since $A_{e}<A$, the nominal surface area, one expects that, for asperity contact $\left\langle\mathrm{F}_{\text {tang }}\right\rangle<A \tau$ and that for $A_{e}=A$ the density, temperature and rate dependence of $\left\langle F_{\text {tang }}>\right.$ is the same as that of $\tau$. In particular, since $\tau$ is proportional to the shear modulus, $G$ (which for pressures less than a significant fraction of the shear modulus, is a linear function of the pressure), the pressure dependence of $\tau$ should be linear for fixed temperature, plastic strain and plastic strain rate. The coefficient of friction, $\mu$, then, which is the ratio of tangential to normal force, is for low and intermediate pressures ${ }^{(1)}$

$$
\mu=\frac{\tau_{0}}{p}\left(1+\alpha \frac{p}{G_{0}}\right) \frac{A_{e}}{A}
$$

Thus for flat interfaces and fixed $\mathrm{T}, \psi$, and $\psi, \mu$ is a decreasing function of pressure in this model. For fixed $T$ and $p$, on the other hand, the flow stress is typically an increasing function of both $\psi$ and $\psi$. and $\mu$ increases. Indeed, at very high strain rates $\tau \propto \dot{\psi}^{\beta}$ with $0<\beta<1$ for many metals.

In summary, when $A_{e}=A$ we may expect that the predicted coefficient of friction is an upper bound to the true value for the rough interface. In the next section we describe a MD simulation technique for an arbitrary interfacial shape and apply it to the flat interface situation. We compare simulations of a copper interface with some of the predictions of the above model.

\section{A MOLECULAR DYNAMICS METHOD FOR THE COEFFICIENT OF FRICTION}

We have carried out a series of MD simulations for systems of 65,000 particles in two dimensions. The boundary conditions which correspond to a typical pin on disk friction experiment are constant normal force (i.e. constant load) and constant tangential velocity. The frictional force is then equal to the external tangential force necessary to keep the tangential velocity constant. We have simulated this situation by dividing our system into two regions, system and reservoir. The reservoir atoms are identical to the system atoms in all respects. In particular, the atom-atom potentials are identical to system particle potentials. However, a body force is applied to the atoms in the reservoir. The applied normal force is a constant and in these simulations it is taken to be that value which summed over reservoir atoms gives the force per unit area equal to the hydrostatic pressure at the system density. A constant tangential force is also applied in such a way that the tangential velocity of reservoir atoms is held constant. This is done in the following way:

Let $x$ be the normal direction, $y$ the tangential direction, and the external tangential force per reservoir atom

$$
F_{e x t}^{y}=\left\langle F^{y}\right\rangle=\left(-\frac{1}{N_{r}}\right) \sum_{i=1}^{N_{r}} F_{i}^{y}
$$

At each time step we compute the total mass in the reservoir,

$$
M_{r}=\sum_{i=1}^{N_{r}} m_{i}
$$

and the total momentum in the $y$ direction for the reservoir atoms,

$$
M_{r}\left\langle\nu^{y}\right\rangle=\sum_{i=1}^{N_{r}} m_{i} \nu_{i}^{y}
$$

Defining $v_{i}^{\prime}$ to be

$$
v_{i}^{\prime} \equiv u_{p}+\left(v_{i}-\left\langle v^{y}\right\rangle\right)
$$

we have that

$$
M_{r}\left(v^{\prime}\right)=\sum_{i=1}^{N_{r}} m_{i} v_{i}^{\prime}=M_{r} u_{p}+\sum_{i=1}^{N_{r}} m_{i} v_{i}^{y}-M_{r}\left(v^{y}\right)
$$


and hence $\left\langle v^{\prime}\right\rangle=u_{p}$.

We solve for reservoir particle motion in the tangential direction by integrating

$$
m_{i} \dot{v}_{i}^{\prime}=F_{i}^{y}+F_{e x t}^{y}
$$

so that $\left\langle\ddot{v}_{i}\right\rangle=0$, consistent with the definition of

$F_{\text {ext }}^{y}$ above. For our two-dimensional simulations we consider initial conditions in which there are equal numbers of atoms above and below the interface with initial velocities fluctuating thermally about $u_{p}$ and $u_{p}$, respectively, in the tangential direction. In these simulations dislocations and sound waves are generated at the interface between the moving materials. We have also included a ramped viscous damping ${ }^{(2)}$ in the reservoir region so that these waves are uniformly absorbed rather than being reflected back toward the interface. Thus, our dynamics more closely models that of an infinite system

\section{SIMULATIONS FOR SLIDING COPPER INTERFACES}

We have performed a series of simulations for sliding copper interfaces for which good density dependent embedded atom model (EAM) potentials exist. The particular copper potential we use is that of Holian et al. (3) which has been chosen to reproduce defect formation energies. This latter ability is particularly important for simulations such as these where plastic deformation and dislocation motion are the driving mechanisms. We use dimensionless units in terms of atomic mass $m$, nearest neighbor distance $r_{0}$ in the $\mathrm{T}=\mathrm{p}=0$ crystal, and bond energy $\varepsilon$. For copper these values are $\mathrm{m}=63.5 \mathrm{amu}, \mathrm{r}_{0}=2.16 \mathrm{~A}, \varepsilon=56 \mathrm{~kJ} / \mathrm{mole}$. The unit of time is expressible in these units as $t_{0}=r_{0} \sqrt{m / \varepsilon}$ which for copper is $t_{0}=2.3010^{-13}$ sec. Our typical simulations were performed for a two-dimensional triangular lattice separated by flat and rough boundaries. The rough boundaries consisted of shifted sine waves, $x=(A+\delta A) \sin (k y+\Delta \phi)$, of a given amplitude $A$ upon which a random amplitude fluctuation $\delta \mathrm{A}$ was imposed. We report here only the studies of the effectively flat interface which was taken to be a single sine wave of amplitude $A=0.5$ and roughness $\delta A=0.01 \mathrm{~A}$ shifted by a phase of $\pi / 4$. (The larger amplitude cases with more waves allowed us to study asperity impacts and deformations.) Initial conditions were average velocities of $-u_{p}$ for $x<0$ and $u_{p}$ for $x>0$. A layer of ten hexagonal cells in the $x$ direction on either side comprised the boundary reservoir in which the average velocity was maintained at $\pm u_{p}$ as described above. The normal force applied was consistent with the equilibrium pressure at the given density and was calculated by runs with $u_{\mathrm{p}} \ll 1$ at a given temperature and density. Initial temperatures were zero and the reservoir was thermostated to maintain a fixed temperature. Typical runs were 300 to 700 $t_{0}$ for 65,000 particles with periodic boundary conditions applied in the y direction.

\section{RESULTS}

A summary of results is given in Table 1 for three densities, $\rho=1.19,1.23$, and 1.27 which correspond to pressures of $0.4,0.8$, and $1.2 \mathrm{GPa}$. Velocities of $\mathrm{u}_{\mathrm{p}}=$ $0.2,0.4$, and 1.0 were considered. These are to be compared with the zero pressure shear wave velocity of 3.0. Because we are limited to rather short time scales with $\mathrm{MD}$, we confine our investigation to the high-velocity regime, $u_{p}>0.1$. We find that the tangential force is an increasing function of density, as predicted by the adhesive model (see Table 2.). The coefficient of friction is a weaker function of density in this regime than the adhesive model would predict. This comparison is not exact, however, since we are not holding $\psi$ and $\psi$ constant in our simulations.

The velocity dependence of these results appears to be in contradiction to a naive interpretation of the adhesive model (Eq. 2). If plastic strain and strainrate increase, then Eq. 2 predicts that $\mu$ would increase along with $\tau_{0}$ at constant $p$. However, we find that $\mu$ is a decreasing function of $u_{p}$ and that above a certain 
velocity $\left(u_{\mathrm{P}} \sim 1\right)$, the temporal behavior of the tangential force changes (see fig. 1). Above this critical velocity, the mechanical response of the underdamped stick-slip system scales with time according to

$$
F_{\text {uang }}(t)=f_{\infty} f(\lambda t)
$$

where $\lambda=h^{-1} \sqrt{f_{\infty} / \rho}, f_{\infty}=F_{\text {tang }}(t \rightarrow \infty)$, and $h$ is the reservoir interface distance. This is shown in fig. 2 . At the same time, the structure of the material near the interface changes. A finite band of thickness $\sim 16 \mathrm{~nm}$ forms, characterized by significant dislocation density. This region is not molten but rather nanocrystalline. The thickness of the band appears to reach an asymptotic value, in contrast to the behavior at lower velocities. This dynamic state, which is reminiscent of those observed by Yoshizawa et al. ${ }^{(4)}$, would be interesting to investigate experimentally,

The present simulations have been done in twodimensions with fully three-dimensional potentials. We are at present extending our work to three dimensions. We expect the qualitative behavior as a function of velocity to remain the same, in particular the formation of an interfacial disordered region above a critical velocity.

TABLE 1. Density, $\rho$, and velocity, $u_{p}$, dependence of the tangential force, $\mathrm{F}_{\text {tang, }}$, and coefficient of friction $\mu$.

\begin{tabular}{llll}
\hline$\rho$ & $\mathbf{u}_{\mathrm{p}}$ & $\left\langle\mathbf{F}_{\text {Lang }}\right\rangle$ & $\mu$ \\
\hline 1.19 & 0.2 & 0.027 & 0.54 \\
& 0.4 & 0.012 & 0.23 \\
& 1.0 & 0.0067 & 0.13 \\
1.23 & 0.2 & 0.039 & 0.33 \\
& 0.4 & 0.030 & 0.25 \\
& 1.0 & 0.0082 & 0.07 \\
1.27 & 0.2 & 0.045 & 0.22 \\
& 0.4 & 0.037 & 0.18 \\
& 1.0 & 0.011 & 0.05 \\
\hline
\end{tabular}

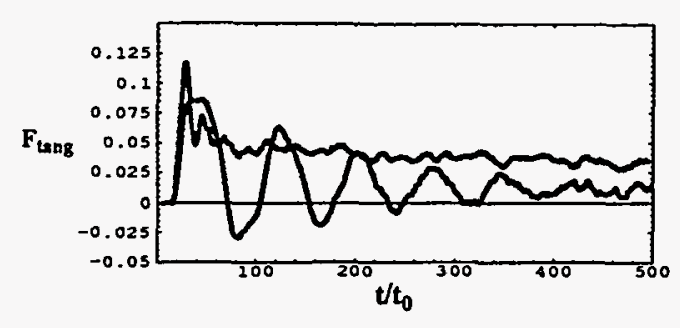

FIGURE 1. Time dependence of the tangential force for $\rho=1.27$. Upper curve: $u_{p}=.04$. Lower curve $u_{p}=1.0$. Curves have been smoothed by averaging over 10 .

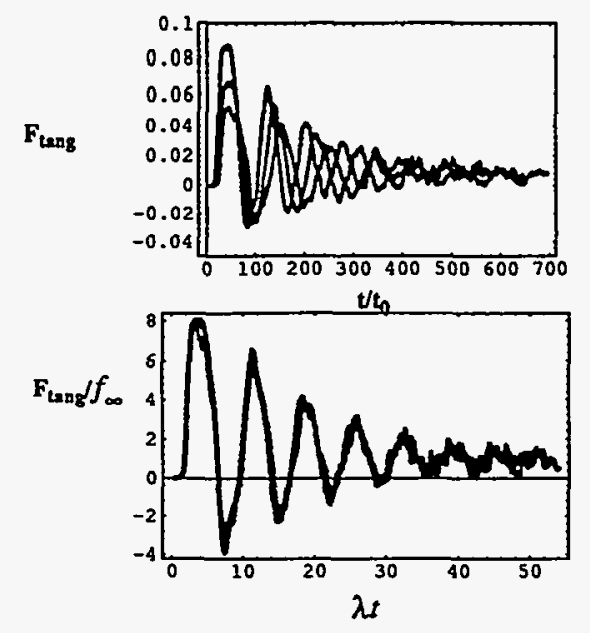

FIGURE 2. Scaling of the tangential force vs. time for $u_{\mathrm{p}}=1.0$. Upper curves (from top) $\rho=1.19,1.23$, and 1.27. Lower curves: scaled curves according Eq. 9. Curves have been smoothed by averaging over 10 to.

\section{REFERENCES}

1. For extremely high pressures, the shear modulus is determined by the dielectric properties of the electron gas and a different law holds.

2. Holian,B.L. and Ravelo,R.,Phys. Rev. 51B,11275-11288(1995).

3. Holian,B.L., et al.Phys Rev.43A,2655 -2661(1991).

4. Yoshizawa,H., et al., Science 259,13051308 (1993).

\section{DISCLAIMER}

This report was prepared as an account of work sponsored by an agency of the United States Government. Neither the United States Government nor any agency thereof, nor any of their employees, makes any warranty, express or implied, or assumes any legal liability or responsibility for the accuracy, completeness, or usefulness of any information, apparatus, product, or process disclosed, or represents that its use would not infringe privately owned rights. Reference herein to any specific commercial product, process, or service by trade name, trademark, manufacturer, or otherwise does not necessarily constitute or imply its endorsement, recommendation, or favoring by the United States Government or any agency thereof. The views and opinions of authors expressed herein do not necessarily state or reflect those of the United States Government or any agency thereof. 